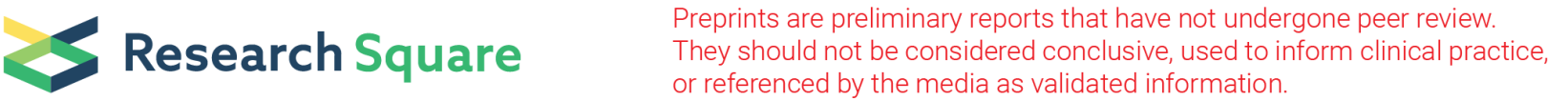 \\ Three novel mutations of microphthalmos identified in two Chinese families
}

\author{
Yating Tang \\ Fudan University Eye Ear Nose and Throat Hospital Department of Ophthalmology \\ Jie Xu \\ Fudan University Eye Ear Nose and Throat Hospital Department of Ophthalmology \\ Tianyu Zheng ( $\nabla$ susu0102@163.com ) \\ Fudan University Eye Ear Nose and Throat Hospital Department of Ophthalmology

\section{Yi Lu} \\ Fudan University Eye Ear Nose and Throat Hospital Department of Ophthalmology
}

\section{Research article}

Keywords: microphthalmos eyes, genetic diagnosis, gene mutation, PXDN, CRYBB2

Posted Date: February 19th, 2020

DOI: https://doi.org/10.21203/rs.2.23917/v1

License: (c) (i) This work is licensed under a Creative Commons Attribution 4.0 International License. Read Full License 


\section{Abstract}

Background: To identify the underlying genetic defect responsible for microphthalmos eyes in two three-generation Chinese families.

Methods: In our study, we screened 425 potential eye disease-related genes of the proband of a three-generation Chinese family diagnosed with microphthalmos using next-generation sequencing-based target capture sequencing. Variants were filtered and analyzed to identify possible disease-causing variants before Sanger sequencing validation.

Results: We enrolled two families with microphthalmos (Family 1: microphthalmos with congenital ocular coloboma and Family 2: simple microphthalmos). Two novel heterozygous mutations, PXDN c.3165C>T (p.Pro1055Pro) and PXDN c.2640C>G (p.Arg880Arg), were found in Family 1, and CRYBB2 c.481G>A (p.Gly161Arg) was found in Family 2, but none of the mutations were found in the unaffected individuals, who were phenotypically normal. Multiple orthologous sequence alignment (MSA) revealed that the CRYBB2 p.Gly161Arg mutation was a deleterious effect mutation.

Conclusions: The three novel mutations found in our study extend our current understanding of the genetic basis of microphthalmos and provide early presymptomatic diagnosis and emphasize the significance of genetic diagnosis of microphthalmos.

\section{Background}

Microphthalmos is a rare, inherited or sporadic congenital eye development defection and is responsible for approximately $3-12 \%$ visual impairment in children. It is characterized by a short axial length ( $A L<20.0-21.0 \mathrm{~mm})$ with thickened choroid and sclera [1, 2], a small anterior chamber depth $(A C D<2.2 \mathrm{~mm})$ and a small cornea (horizontal corneal diameter $<11 \mathrm{~mm}$ ) [3]. According to the anatomical difference and American Society of Cataract and Refractive Surgery (ASCRS) cataract clinical committee, microphthalmos can be divided into four types: simple microphthalmos (or nanophthalmos), microphthalmos with other congenital ocular coloboma, relative anterior microphthalmos (RAM) and axial high hyperopia $[2,4]$.

Genetic alterations are now a major cause of microphthalmos. The hereditary mode of nanophthalmos is autosomal dominant in many patients and sporadic in others [5,6]. As a developmental eye disease, most of the related genes involved in microphthalmos are also ocular development-associated genes, including SOX2, OTX2, PAX6, and GJA8. Although over 30 genes have been implicated in eye development, we are currently able to explain the genetic bases of these defects in less than half of patients [5]. Moreover, recent studies on microphthalmia mainly rely on clinical observation, and novel related genes and mutations in microphthalmos have rarely been explored.

Hence, in our study, we studied the novel mutation genes of microphthalmos in two Chinese families (Family 1: microphthalmos with congenital ocular coloboma and Family 2: simple microphthalmos) and reported three novel mutations. Hopefully, our study will be helpful for microphthalmos prediction, prevention and molecular treatment.

\section{Methods}

\section{Subjects}


To make a precise diagnosis, we performed targeted next-generation sequencing (NGS) of microphthalmos-related genes. All featured participants provided written consent to participate, and the parents/guardians of any minors that participated provided written consent to participate on their behalf. The study was approved by the ethics committee of Eye and ENT Hospital of Fudan University and was conducted according to the principles of the Declaration of Helsinki.

Our study involved two family members from three generations who underwent detailed history ophthalmic examination, including best corrected visual acuity (BCVA) testing, slit lamp biomicroscopy, IOL master 500 (Carl Zeiss Meditec, Germany), dilated fundus examination, B scan, and SD-OCT (Spectralis HRA + OCT, Heidelberg, Engineering, Inc., Heidelberg, Germany). Family and medical history, including age of onset and other related clinical manifestations, was obtained. Blood samples were collected from the peripheral blood and stored at $4{ }^{\circ} \mathrm{C}$ before further analysis.

\section{Genetic analyses}

We extracted the genomic DNA from the family from peripheral blood on Feb. 2018 according to the manufacturer's standard procedure using the QIAamp DNA Blood Midi Kit (Qiagen, Hilden, Germany) [7]. A capture panel (NimbleGen, Madison, USA) of microphthalmos genes has been previously designed and assessed by our group. The capture panel comprised all exons together with the flanking exon and intron boundaries ( \pm 15 bp) of 425 genes that are most frequently involved in common inherited eye diseases (the capture probes were custom designed and produced by BGI).

The library was enriched by array hybridization according to a previously published procedure, followed by elution and postcapture amplification. Then, qualification and NGS targeted sequences were further analyzed on the Illumina HiSeq 2000 platform (Illumina, Inc., San Diego, CA, United States) in collaboration with BGI-Shenzhen (Shenzhen, Guangdong, China) as previously reported $[8,9]$. The filtered sequencing was then aligned to the human genome reference (hg19) using the Burrows Wheeler Aligner (BWA) Multi-Vision software package [10]. After alignment, the output files were used to perform sequencing coverage and depth analysis of the target region, single-nucleotide variants (SNVs) and INDEL calling.

We used the following 4 databases to test annotation of all identified variants with minor allele frequency (MAF) > $0.1 \%$ to eliminate benign variants: dbSNP137, HapMap Project, 1000 Genomes Projectand Exome Variant Server. Finally, the variant prioritizations were performed, combining total depth, quality score, MAF, potential deleterious effect and the existence of mutation reports in common databases such as the Human Gene Mutation Database (HGMD), Online Mendelian Inheritance in Man (OMIM). All mutations and potential pathogenic variants were validated using conventional Sanger sequencing or a second spectrum sequencing method. Segregation analysis was performed if DNA from family members was available.

\section{Results}

\section{Clinical examination and pedigree analysis}

A total of 17 members from two three-generation families (Fig. 1) were included in the study. The clinical information of the four affected members is listed in Table 1. The proband of Family 1 (P5), a 29-year-old woman, has suffered from poor vision in both eyes since birth. Ophthalmic examination revealed a BCVA HM in both eyes. The axial length measurement showed the extreme short axial length (OD: $18.36 \mathrm{~mm}, \mathrm{OS}: 19.83 \mathrm{~mm}$ ). We also 
found an extremely small cornea diameter (OD: $6.5 \mathrm{~mm}$, OS: $7.2 \mathrm{~mm}$ ) after corneal diameter evaluation. Fundus examination and B scan both revealed severe choroid colomoba (Fig. 2a). After careful examination of her son, we found that P5 and her son (P9) suffered from the same eye disease: microphthalmos with congenital ocular coloboma and congenital cataract (Fig. 2b).

Table 1

Clinical characteristics of the four affected patients

\begin{tabular}{|c|c|c|c|c|}
\hline \multirow[t]{2}{*}{ Patients } & \multicolumn{2}{|c|}{ Family $1(D L L)$} & \multicolumn{2}{|c|}{ Family 2 (FBF) } \\
\hline & Patient 5 & Patient 9 & Patient 4 & Patient 7 \\
\hline Age & 29 & 2 & 31 & 7 \\
\hline BCVA (OD/OS) & $\mathrm{HM} / \mathrm{HM}$ & / & $\mathrm{FC} / \mathrm{FC}$ & $0.05 / F C$ \\
\hline Axial length (mm)(OD/OS) & $18.36 / 19.83$ & $15.77 / 15.62$ & $16.99 / 16.02$ & $16.57 / 16.34$ \\
\hline $\begin{array}{l}\text { Anterior chamber depth } \\
\text { (OD/OS) }\end{array}$ & $1.07 / 1.21$ & / & / & / \\
\hline $\begin{array}{l}\text { Cornea diameter }(\mathrm{mm}) \\
\text { (OD/OS) }\end{array}$ & $6.5 / 7.2$ & $6.4 / / 6.1$ & $9.7 / 9.0$ & $9.3 / 9.1$ \\
\hline Intraocular pressure (OD/OS) & $8.2 / 13.9$ & / & / & / \\
\hline B scan & \multicolumn{2}{|c|}{ choroid coloboma } & \multicolumn{2}{|l|}{ normal } \\
\hline Diagnosis & \multicolumn{2}{|c|}{$\begin{array}{l}\text { microphalmos with congenital ocular } \\
\text { coloboma }\end{array}$} & \multicolumn{2}{|c|}{ simply microphthalmos } \\
\hline
\end{tabular}

The proband of Family 2 (P4), a 31-year-old woman, has suffered from progressive decreased vision in both eyes for over 10 years. Ophthalmic examination also revealed extremely short eyes (axial length: OD: 16.99 mm, OS: $16.02 \mathrm{~mm}$ ) and small cornea (OD: $9.7 \mathrm{~mm}$, OS: $9.0 \mathrm{~mm}$ ) with congenital cataract. Fundus examination and a $B$ scan showed moderate normal fundus. After careful examination of her daughter (P7), P4 and her daughter were diagnosed as the same disease: simple microphthalmos.

\section{Genetic analyses}

We performed a targeted NGS approach on the probands (P5 of Family 1 and P4 of Family 2). The targeted gene length was $1,106,466$ bp of 425 genes, and the mean depth of the target region was 170.45 with $99.95 \%$ coverage. After the data acquisition and bioinformation analysis, two novel heterozygous mutations, PXDN c.3165C > T (p.Pro1055Pro) and PXDN c.2640C > G (p.Arg880Arg), were found in the proband (P5) of Family 1 (Fig. 3 and Table 2), and CRYBB2 c.481G > A (p.Gly161Arg) was found in Family 2 (Fig. 4 and Table 2). These mutations, PXDN c.3165C > T (p.Pro1055Pro), PXDN c.2640C > G (p.Arg880Arg) and CRYBB2 c.481G > A (p.Gly161Arg), were extremely rare in the control population, with frequencies of $0.0082,0.0082$ and 0 in the 1000 Genomes Project, respectively. Moreover, the three mutations in the SNV database of over 200 Chinese populations were less than 0.01 . 
Table 2

Mutations identified in family 1 and family 2

\begin{tabular}{|llllllll|}
\hline Family & Gene & $\begin{array}{l}\text { NCBI } \\
\text { reference } \\
\text { sequence }\end{array}$ & $\begin{array}{l}\text { Nucleotide } \\
\text { change }\end{array}$ & $\begin{array}{l}\text { Amino acid } \\
\text { substitution }\end{array}$ & $\begin{array}{l}\text { Chromosomal } \\
\text { location }\end{array}$ & $\begin{array}{l}\text { Gene } \\
\text { subregion }\end{array}$ & $\begin{array}{l}\text { Allele } \\
\text { status }\end{array}$ \\
\hline $\begin{array}{l}\text { Family } \\
1\end{array}$ & PXDN & NM_012293 & $\begin{array}{l}\text { C.3165C }> \\
\text { T }\end{array}$ & p.Pro1055Pro & chr2:1652387 & EX17/CDS17 & Het. \\
\hline & PXDN & NM_012293 & $\begin{array}{l}\text { C.2640C }> \\
\text { G }\end{array}$ & p.Arg880Arg & chr2:1652912 & EX17/CDS17 & Het. \\
$\begin{array}{l}\text { Family } \\
2\end{array}$ & CRYBB2 & NM_000496 & C.481G >A & p.Gly161Arg & chr22:25627602 & EX6/CDS5 & Het. \\
\hline
\end{tabular}

Sanger sequencing was then performed to validate the variants in Family 1. The proband's son (P9) of Family 1 carried the same heterozygous mutations (c.3165C > T and c.2640C > G) of the PXDN gene (Fig. 3 and Table 2). However, the two mutations were not found in the proband's parents. However, the two mutations did not lead to amino acid changes.

We used the spectrum sequencing method to validate the variant in Family 2 after the negative result of the Sanger sequencing method. The proband's daughter (P7) in Family 2 carried the same heterozygous mutation (c.481G > A) of the CRYBB2 gene. The mutation was not found in the proband's parents. Multiple orthologous sequence alignment (MSA) using Polyphen revealed that CRYBB2 and its subsequent sequences were highly conserved amino acids across different species (Fig. 5), suggesting that the mutation may lead to a deleterious effect.

\section{Discussion}

As a high-throughput, low-cost and high-efficiency sequencing technology, NGS technology has been widely used in clinics to identify many rare diseases $[7,11]$. The identified mutations and genes have become an important tool to explore potential genetic etiology and guide appropriate treatment for eye diseases [7, 12]. In our study, we comprehensively screened 425 genes involved in common, inherited eye diseases and successfully identified 3 potentially causative mutations for microphthalmos, PXDN c.3165C > T (p.Pro1055Pro), PXDN c.2640C > G (p.Arg880Arg) and CRYBB2 c.481G > A (p.Gly161Arg), in two Chinese families. Based on the mutation analysis and the clinical measurement, we concluded that these three novel mutations could provide early presymptomatic diagnoses and emphasize the significance of the genetic diagnosis of microphthalmos.

The human CRYBB2 (crystalline beta B2) gene is located on chromosome 22q and is encoded in 7 exons. The CRYBB2 gene is a dimer at low concentrations but can form oligomers under physiological conditions. The CRYBB2 gene has contributed to the identical human lens crystalline protein-protein binding [13, 14], structural constituent of human lens [15] and structural molecule activity [16]. Multiple studies have revealed that CRYBB2 gene mutations (including p.Q155X [17-19], p.E167X [20]) were associated with congenital autosomal dominant cataracts. In our study, we also found a CRYBB2 novel mutation (p.Gly161Arg) in simply microphthalmos eyes in Family 2. The proband and her daughter shared the same mutation of the CRYBB2 gene, which adhered to the dominant heredity as previous papers. Furthermore, MSA using Polyphen revealed that CRYBB2 and its subsequent sequences were highly conserved amino acids across different species, suggesting that the CRYBB2 p.Gly161Arg mutation was a deleterious mutation. To the best of our knowledge, the CRYBB2 p.Gly161Arg mutation was 
originally reported in this study. However, the proband and her daughter had microphthalmos and congenital cataract, and whether the CRYBB2 p.Gly161Arg mutation is a causative mutation of microphthalmos or the congenital cataract needs further in vitro study.

The human PXDN geneis located on chromosome 2p and is encoded in 24 exons. The gene is expressed in corneal epithelium and is secreted into the extracellular matrix. Mutations in the PXDN gene were associated with congenital recessive corneal opacification and other ocular anomalies as well as microphthalmia and anterior segment dysgenesis. Choi et al [21] reported PXDN Tyr398Thrfs*40 and PXDN GIn316Pro mutations in three families with congenital cataracts, microcornea, sclerocornea and developmental glaucoma. The defective PXDN gene has been shown to impair sulfilimine bond formation in collagen IV, a constituent of the basement membrane, implying that eye defects result from the loss of basement membrane integrity in the developing eye. In our study, we also found that PXDN gene mutations c.3165C > T (p.Pro1055Pro) and c.2640C > G (p.Arg880Arg) in microphthalmos with congenital ocular coloboma eyes. The two mutations have not been previously reported. Furthermore, the heredity model was prone to be autosomal dominant in Family 1, which implied that the PXDN gene mutations c.3165C > T (p.Pro1055Pro) and c.2640C > G (p.Arg880Arg) might be different from the other PXDN gene mutations. Although the two mutations did not result in amino acid changes, we also concluded that PXDN sequencing should be considered in microphthalmos with anterior segment dysgenesis.

To the best of our knowledge, this study used the first NGS-based assay specifically designed for the confirmation and early diagnosis of microphthalmos in two Chinese families' pedigrees reported to date. In addition, we found 3 potentially causative mutations for microphthalmos, PXDN c.3165C > T (p.Pro1055Pro), PXDN c.2640C > G (p.Arg880Arg) and CRYBB2 c.481G > A (p.Gly161Arg), that are likely responsible for microphthalmos. These genetic mutation patterns are novel, and the functions and interactions of the PXDN and CRYBB2 genes should be further investigated. This study not only provides a guide to the attending clinician on the management and prognosis of the patient but also extends the phenotypic spectrum of PXDN- and CRYBB2- associated microphthalmos and enhances our current understanding of the genetic basis of microphthalmos.

\section{Abbreviations}

MSA:Multiple orthologous sequence alignment; AL:Axial length; ACD:Anterior chamber depth; RAM:Relative anterior microphthalmos; NGS:Next-generation sequencing; BCVA:Best corrected visual acuity; SNVs:Single-nucleotide variants; MAF:Minor allele frequency

\section{Declarations}

\section{Acknowledgments}

Not applicable.

\section{Authors' contributions}

Study concept and design ( $T Z, Y L)$; data collection (YT, JX); analysis and interpretation of data (YT, JX); writing the manuscript (YT); critical revision of the manuscript (JX, TZ, YL); administrative, technical, or material support (TZ, $\mathrm{YL})$; supervision (TZ,YL). All authors have read and approved the manuscript.

\section{Funding}


This work was financially supported by the grants for Natural Science Foundation of China (NSFC 81670835 and NSFC 81600719), the Shanghai Natural Science Foundation of China (Grant No. 19ZR1408600), the Shanghai Science and Technology Commission (11231200602) and the Visual Impairment and Reconstruction Key Laboratory of Shanghai (12DZ2260500). Role of the funding: collection, analysis of data and in writing the manuscript.

\section{Availability of data and materials}

The datasets used and/or analysed during the current study are available from the corresponding author on reasonable request.

\section{Ethics approval and consent to participate}

This study was approved by the ethics committee of the Eye and Ear, Nose, and Throat (ENT) Hospital of Fudan University and was conducted according to the principles of the Declaration of Helsinki. The written informed consent was obtained from the patient.

\section{Consent for publication}

Not applicable.

\section{Competing interests}

The authors declare that they have no competing interests. Dr. Lu is a member of the editorial board of this journal.

\section{Author details}

${ }^{1}$ Department of Ophthalmology, Eye and Ear, Nose, and Throat Hospital, Fudan University, 83 Fenyang Rd., Shanghai 200031, China. ²Eye Institute, Eye and Ear, Nose, and Throat Hospital, Fudan University, Shanghai, China.

${ }^{3}$ Key Laboratory of Myopia, Ministry of Health, Shanghai, China. ${ }^{4}$ Shanghai Key Laboratory of Visual Impairment and Restoration, Shanghai, China.

\section{References}

1. Verma AS, Fitzpatrick DR. Anophthalmia and microphthalmia. Orphanet J Rare Dis. 2007;2:47.

2. Hoffman RS, Vasavada AR, Allen QB, Snyder ME, Devgan U, Braga-Mele R. Cataract surgery in the small eye. J Cataract Refract Surg. 2015;41(11):2565-2575.

3. Nihalani BR, Jani UD, Vasavada AR, Auffarth GU. Cataract surgery in relative anterior microphthalmos. Ophthalmology. 2005;112(8):1360-1367.

4. Zheng T, Chen Z, Xu J, Tang Y, Fan Q, Lu Y. Outcomes and Prognostic Factors of Cataract Surgery in Adult Extreme Microphthalmos With Axial Length $<18 \mathrm{~mm}$ or Corneal Diameter $<8 \mathrm{~mm}$. Am J Ophthalmol. 2017;184:84-96.

5. Plaisancié J, Ceroni F, Holt R, Zazo Seco C, Calvas P, Chassaing N, Ragge NK. Genetics of anophthalmia and microphthalmia. Part 1: Non-syndromic anophthalmia/microphthalmia. Hum Genet. 2019.

6. Slavotinek A. Genetics of anophthalmia and microphthalmia. Part 2: Syndromes associated with anophthalmia-microphthalmia. Hum Genet. 2018. 
7. Ma X, Guan L, Wu W, Zhang Y, Zheng W, Gao YT, Long J, Wu N, Wu L, Xiang Y, et al. Whole-exome sequencing identifies OR2W3 mutation as a cause of autosomal dominant retinitis pigmentosa. Sci Rep. 2015;5:9236.

8. Chen X, Liu X, Sheng X, Gao X, Zhang X, Li Z, Li H, Liu Y, Rong W, Zhao K, et al. Targeted next-generation sequencing reveals novel EYS mutations in Chinese families with autosomal recessive retinitis pigmentosa. Sci Rep. 2015;5:8927.

9. Chen X, Zhao K, Sheng X, Li Y, Gao X, Zhang X, Kang X, Pan X, Liu Y, Jiang C, et al. Targeted sequencing of 179 genes associated with hereditary retinal dystrophies and 10 candidate genes identifies novel and known mutations in patients with various retinal diseases. Invest Ophthalmol Vis Sci. 2013;54(3):2186-2197.

10. Li H, Durbin R. Fast and accurate short read alignment with Burrows-Wheeler transform. Bioinformatics. 2009;25(14):1754-1760.

11. Patel A, Hayward JD, Tailor V, Nyanhete R, Ahlfors H, Gabriel C, Jannini TB, Abbou-Rayyah Y, Henderson R, Nischal KK, et al. The Oculome Panel Test: Next-Generation Sequencing to Diagnose a Diverse Range of Genetic Developmental Eye Disorders. Ophthalmology. 2019;126(6):888-907.

12. Plaisancie J, Calvas P, Chassaing N. Genetic Advances in Microphthalmia. J Pediatr Genet. 2016;5(4):184-188.

13. Xu J, Zhao WJ, Chen XJ, Yao K, Yan YB. Introduction of an extra tryptophan fluorophore by cataractassociating mutations destabilizes betaB2-crystallin and promotes aggregation. Biochem Biophys Res Commun. 2018;504(4):851-856.

14. Liu BF, Liang JJ. Domain interaction sites of human lens betaB2-crystallin. J Biol Chem. 2006;281(5):26242630 .

15. Chambers C, Russell P. Sequence of the human lens beta B2-crystallin-encoding cDNA. Gene. 1993;133(2):295299.

16. Zhao WJ, Xu J, Chen XJ, Liu HH, Yao K, Yan YB. Effects of cataract-causing mutations W59C and W151C on betaB2-crystallin structure, stability and folding. Int J Biol Macromol. 2017;103:764-770.

17. Li FF, Zhu SQ, Wang SZ, Gao C, Huang SZ, Zhang M, Ma X. Nonsense mutation in the CRYBB2 gene causing autosomal dominant progressive polymorphic congenital coronary cataracts. Mol Vis. 2008;14:750-755.

18. Ching YH, Yeh JI, Fan WL, Chen KC, Yeh MC, Woon PY, Lee YC. A CRYBB2 mutation in a Taiwanese family with autosomal dominant cataract. J Formos Med Assoc. 2019;118 (1 Pt 1):57-63.

19. Messina-Baas O, Gonzalez-Garay ML, Gonzalez-Huerta LM, Toral-Lopez J, Cuevas-Covarrubias SA. Whole Exome Sequencing Reveals a Mutation in CRYBB2 in a Large Mexican Family with Autosomal Dominant Pulverulent Cataract. Mol Syndromol. 2016;7(2):87-92.

20. Zhou Y, Zhai Y, Huang L, Gong B, Li J, Hao F, Wu Z, Shi Y, Yang Y. A Novel CRYBB2 Stopgain Mutation Causing Congenital Autosomal Dominant Cataract in a Chinese Family. J Ophthalmol. 2016;2016:4353957.

21. Choi A, Lao R, Ling-Fung Tang P, Wan E, Mayer W, Bardakjian T, Shaw GM, Kwok PY, Schneider A, Slavotinek A. Novel mutations in PXDN cause microphthalmia and anterior segment dysgenesis. Eur J Hum Genet. 2015;23(3):337-341.

\section{Figures}



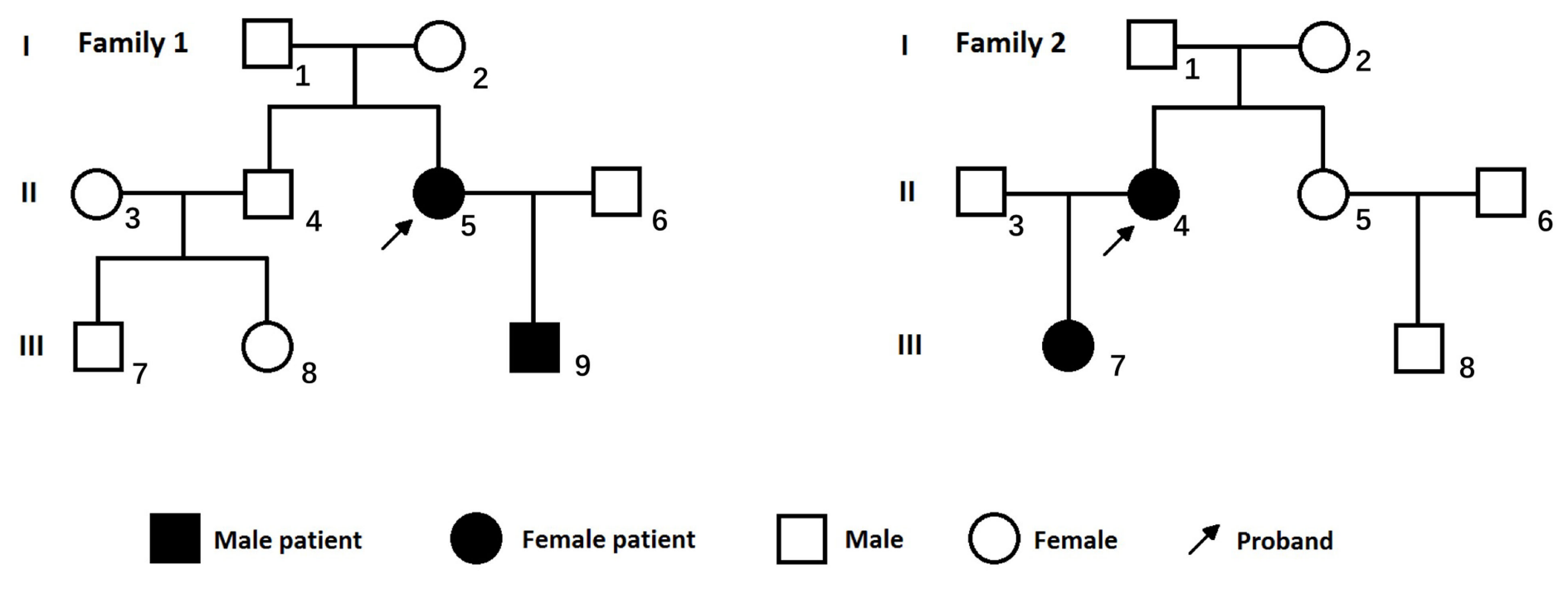

Figure 1

Proband of the two families. 


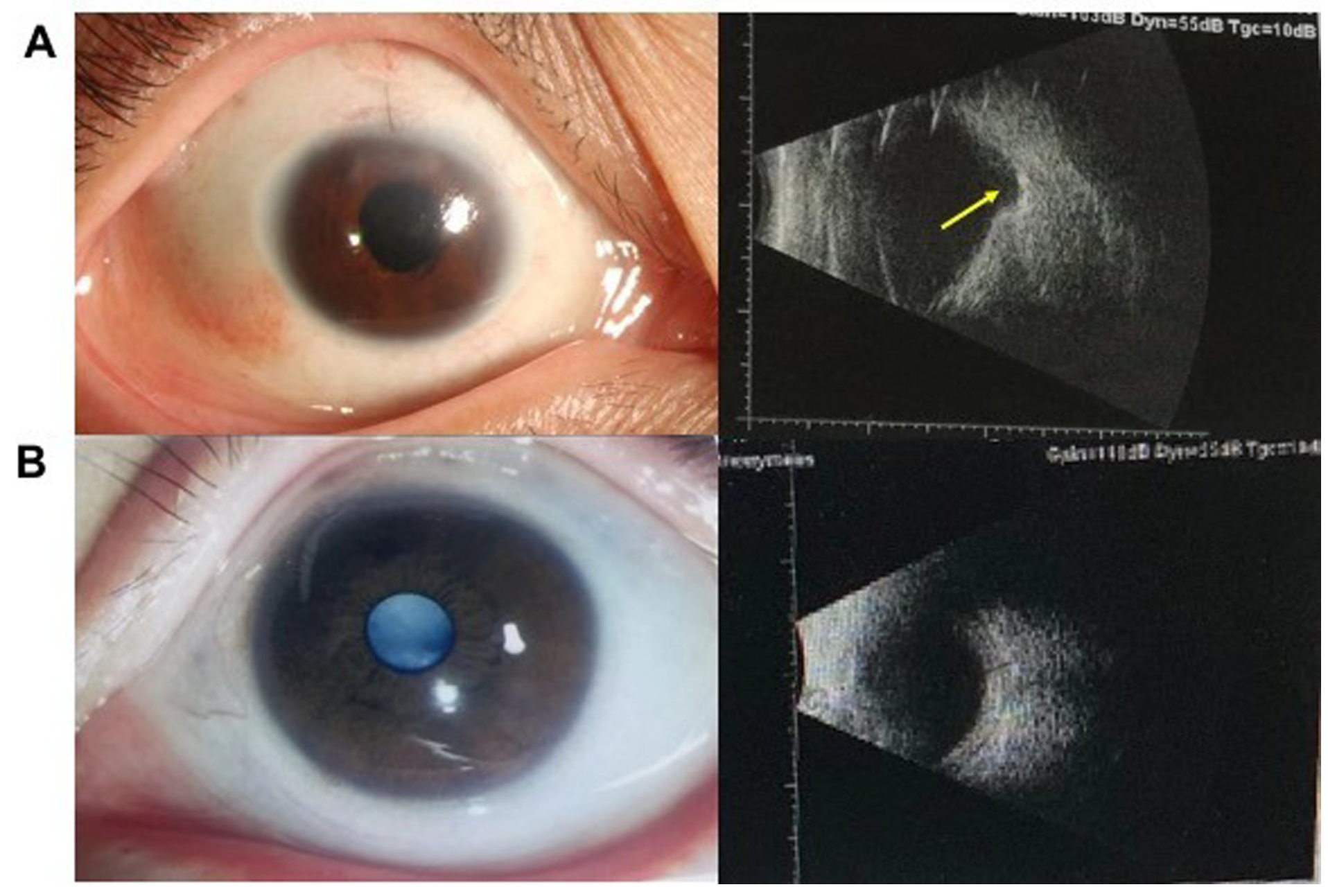

\section{Figure 2}

a Slit lamp photograph (left) and B scan (right) of proband (P5) of Family 1. The yellow arrow shows the severe choroid colomoba. b Slit lamp photograph (left) and B scan (right) of proband (P4) of Family 2. 


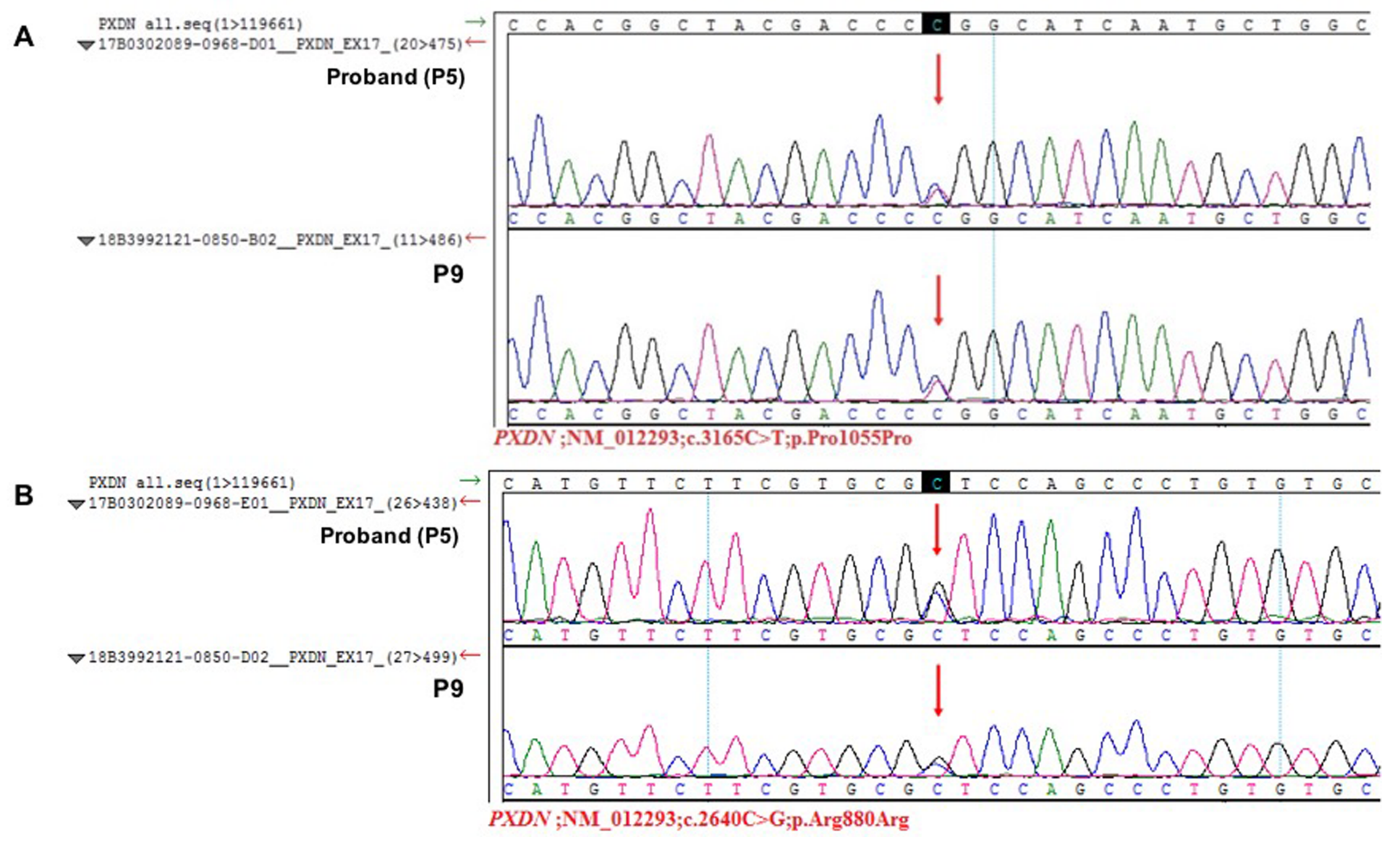

Figure 3

PXDN gene mutations found in Family 1 .

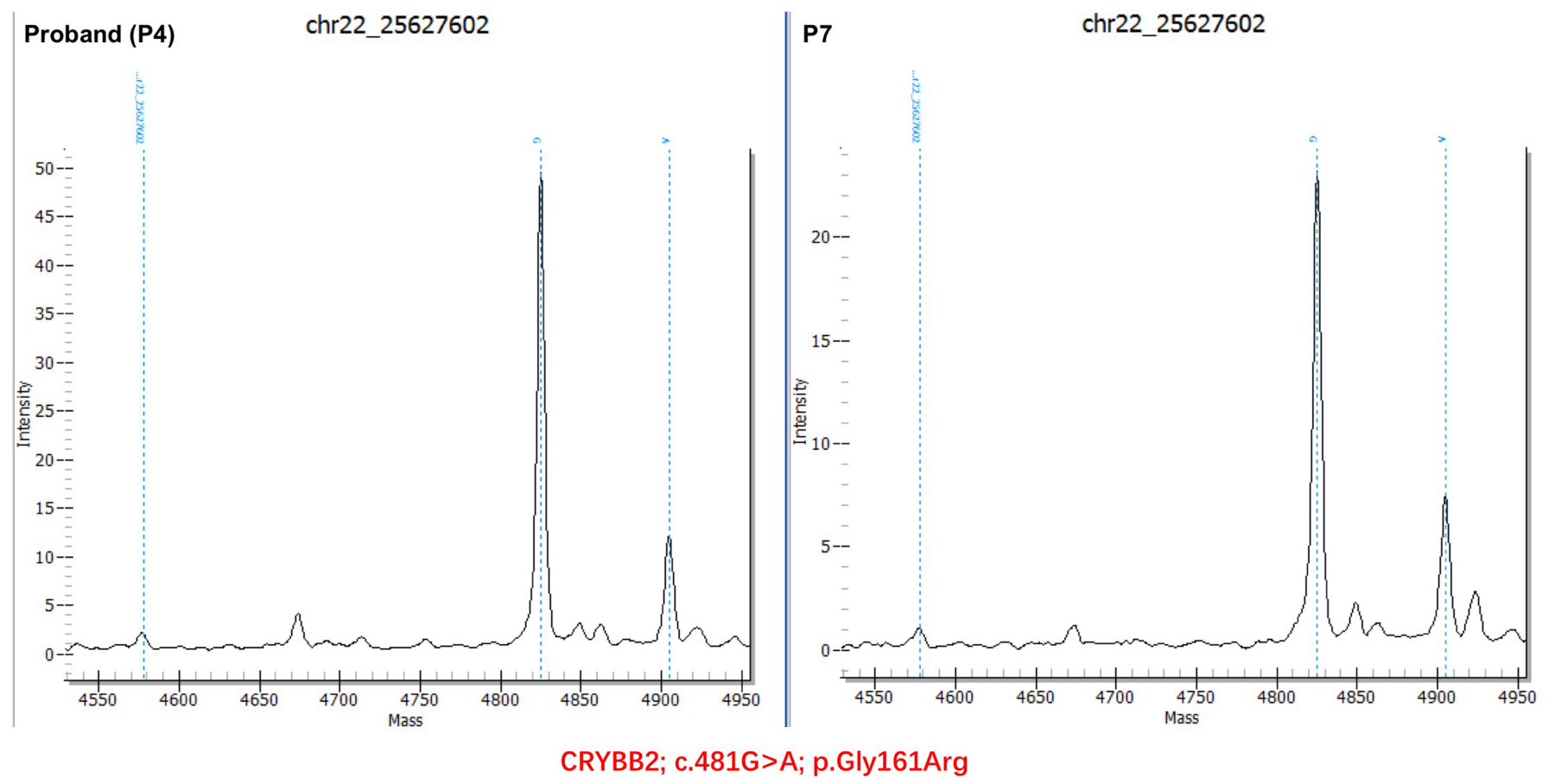


Figure 4

CRYBB2 gene mutation found in Family 2.

p.Gly161Arg

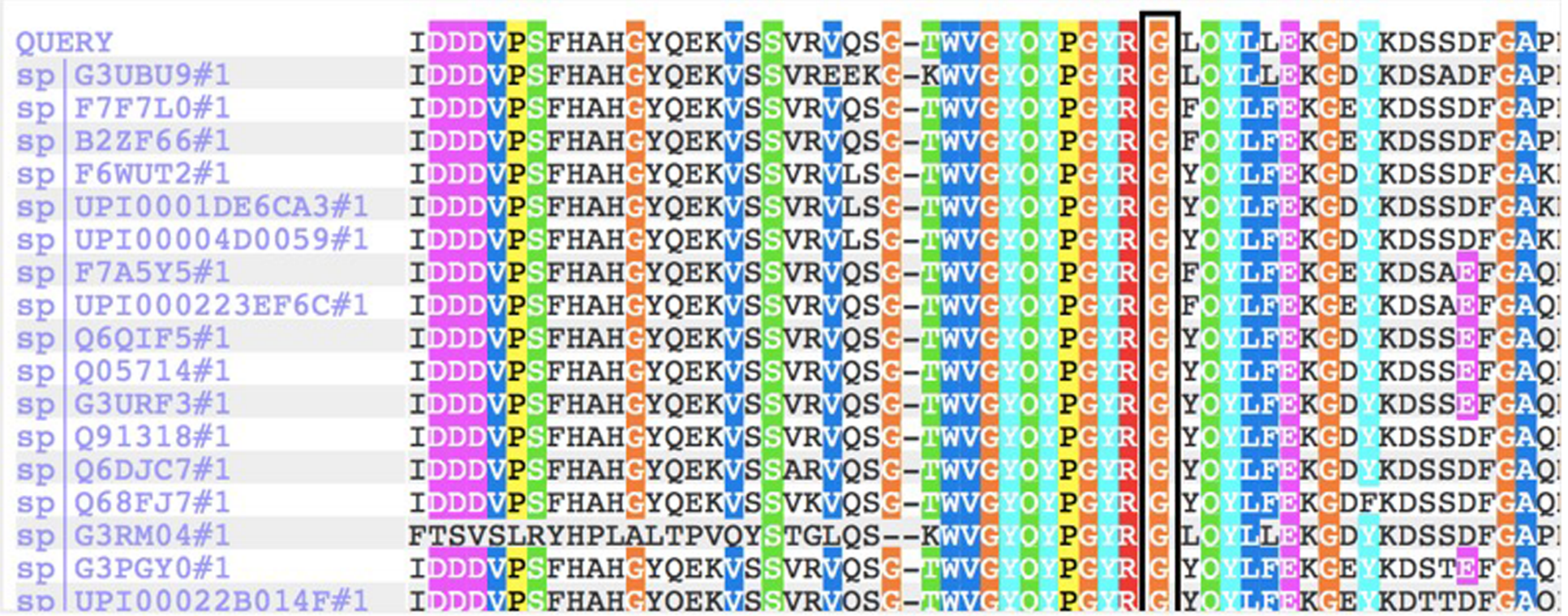

Figure 5

Multiple orthologous sequence alignment (MSA) for CRYBB2 p.Gly161Arg mutation. 